\title{
A Retinal Blood Vessel Segmentation Approach Based on Top-hat Transformation
}

\author{
Weihua Wang ${ }^{1,2,3, a}$, Wenyuan $\mathrm{Wu}^{1}$, Jiao Yin $^{3}$ \\ ${ }^{1}$ Chongqing Key Laboratory of Automated Reasoning and Cognition, Chongqing Institute of Green and \\ Intelligent Technology, Chinese Academy of Sciences, Chongqing, 400714, China \\ ${ }^{2}$ School of Computer and Control Engineering, University of Chinese Academy of Sciences, Beijing, 100049, \\ China \\ ${ }^{3}$ School of Software Engineering, Chongqing University of Arts and Sciences, Yongchuan, Chongqing, \\ 402160, China \\ a2002ww@163.com
}

Keywords: Double threshold, Blood Vessel Segmentation, Fundus Image

\begin{abstract}
The analysis of retinal blood vessel plays an important role in aided diagnosis, segmenting blood from retinal fundus images is the first operation. This paper proposes a novel approach for retinal blood vessel automatic segmentation. First, the top-hat transformation is used for blood vessel enhancement. Second, using double-threshold to segment the vessels from the enhanced fundus image, the high threshold is used for main vessel segmentation, and the low threshold is used for finer vessel segmentation. Third, fusing the finer blood vessels into main blood vessels. The experiment is tested in public fundus image database STARE. The result of the experiment shows that the method obtains good visual effect.
\end{abstract}

\section{Introduction}

Retinal fundus vessel analysis plays an important role in diagnosis of various pathologies, for example macular degeneration and diabetic retinopathy etc. Segmentation of vessel tree is the first step in retinal image analysis. The manual vessel segmentation of retinal image is expensive and time-consuming, the automatic segmentation algorithm is thus very important.

Several methods have been presented for the vessel segmentation in retinal fundus images, these methods can be divided into two categories: supervised and unsupervised methods [1, 2].

Unsupervised method does not need training. Match filter technology is a class of unsupervised vessel segmentation methods, Chaudhuri et al.[3] proposed an 2-D matched filter to enhance and segment retinal image blood vessels. Soares et al.[4] introduced a Gaobr filter method to enhanced retinal blood vessel technology. However, most of the unsupervised methods should segment major vessel, and does not extract thinner vessel accurately.

Supervised methods need manual standard retinal vessel image for training, Marin et al. [5] introduced a supervised technology that using gray level and moment invariants-based features to segment blood vessel. You et al. [6] proposed a radial projection based semi-supervised algorithm to extract the retinal blood vessels in an image. In general, the vessel segmentation performance of the supervised technology is better than that of unsupervised technology, and obtain good visual effect for retinal images. However, the training of the supervised method need manual segmented image, and the manual segmentation is time-expensive.

To counter the above problem, a new unsupervised retinal segmentation method based on top-hat transformation is proposed in this paper. This method use morphology logical to enhance and use double threshold to extract the blood vessel. 


\section{Proposed method}

The proposed method includes three stages: preprocessing, vessel enhancement and retinal vessel segmentation. The aim of preprocessing stage is to obtain gray image and remove noise. Enhancing the contrast between the vessel and background in an image is the basic task of the stage in the vessel enhancement stage. The key process in this method is to segment the vessel from its background. The detailed frame diagram of this proposed algorithm is shown in Fig. 1.

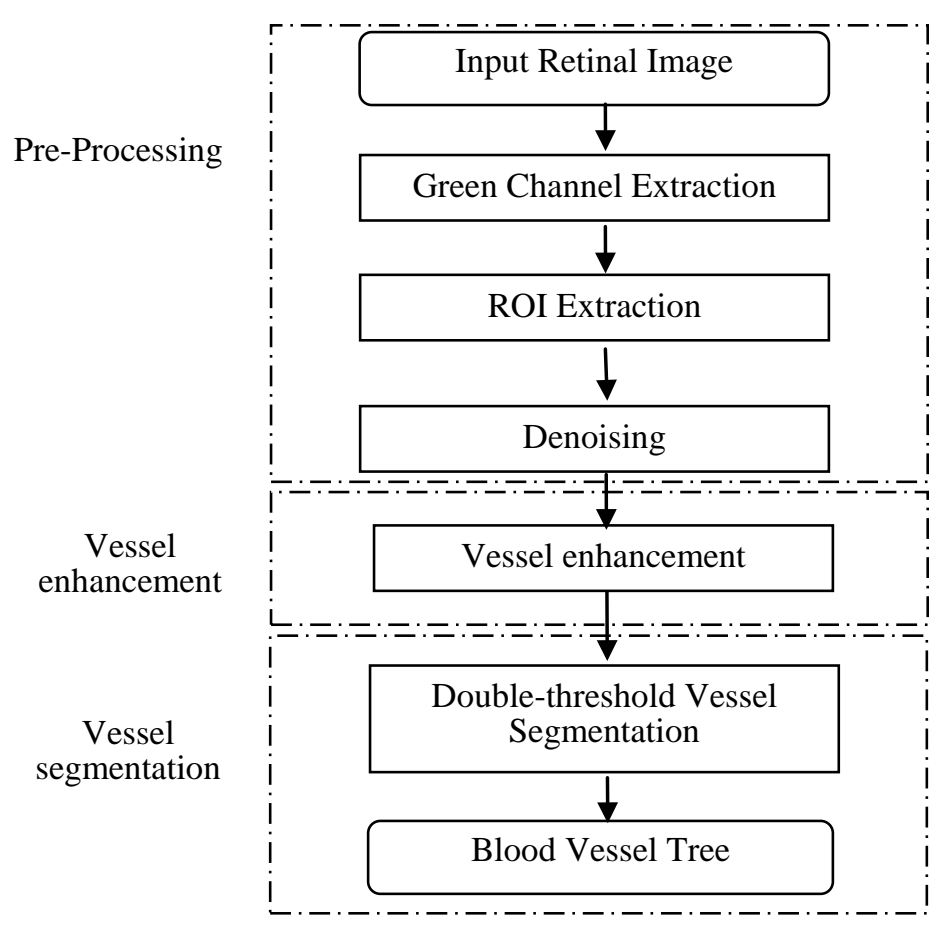

Fig. 1 The Frame diagram of the new method.

\subsection{Pre-processing}

As we know that a retinal image is a color image. In general, people always analysis the features in a gray image, and the green component has the best contrast between the vessel and background in the components of a retinal image. Therefore, the green channel is selected for the post processing in this method. At the same time, the outer region of the field-of-view (FOV) influences the contrast and segmentation of vessels, this method extracts the region of interest (ROI) by the FOV-mask of a retinal. Moreover, in order to remove the isolated noise and improve the vessel segmentation accuracy, a smooth method is used in this method, here, Gaussian filter is selected, which is a 2-D convolution operation.

\subsection{Enhancement}

During the fundus image processing, retinal structures with high intensity overlap the non-vascular and decrease the visibility of thin vessels with low contrast. In addition, the retinal optic disc, fovea, hemorrhages, hard exudate and other pathological changes present complicate should be considered, especially the brightness region of optic disc and hard exudate decrease the contrast in the whole image.

In this study, the top-hat transformation of mathematical morphology is proposed to remove the high brightness region and to improve the contrast between the vessel and background. The opening operation can remove the brightness details with small structure element and retain the gray of the other region. On the contrary, the closing operation can remove the darkness with small structure element and retain the gray of the other region. Therefore, the top-hat filter is designed as follows. First, an input image is processed by opening operation to remove the regions with small area brightness and obtain the regions with large area brightness, that is, obtain the hard exudate and the 
optic disc in the fundus image. Second, the error between the original image and the image processed by opening operation will obtain the filter image, which can remove the regions with large area brightness.

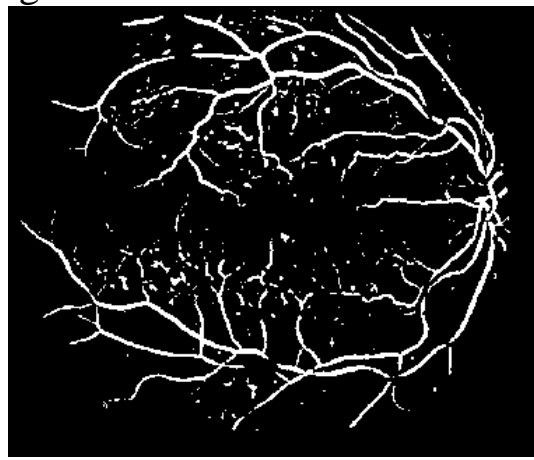

(a) ratio $=8.2 \%$

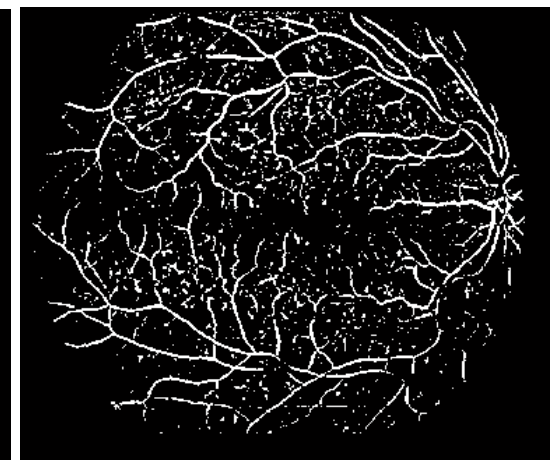

(b) ratio $=9.4 \%$

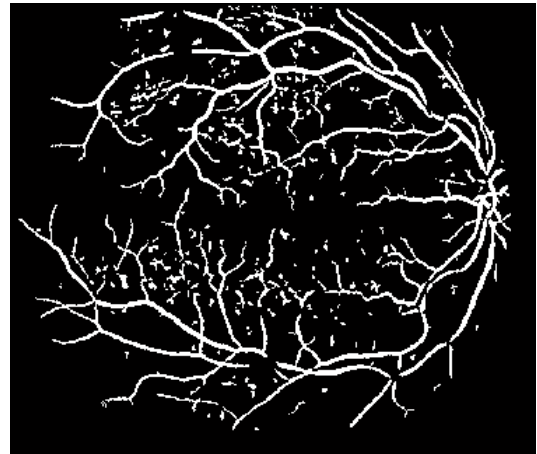

(c) fusion of (a) and (b)

Fig. 2 Different threshold has different vessel

\subsection{Vessel segmentation}

The image enhanced by top-hat transformation in the above step is a gray image, in order to obtain the vessel tree, some method should be used for binary-conversion. This study propose a double-threshold method to extract the vessel. The threshold is calculated by the ration of the vessel pixels in a retinal image. Different threshold has different vessel, this is shown in Fig. 2. The high threshold extracts the less vessels and less noise, and the low threshold extracts more vessels and noise. According to the connection of vessel, the low threshold vessels are tracked by the high threshold vessels, lots of noise is removed, and more vessel are extracted.

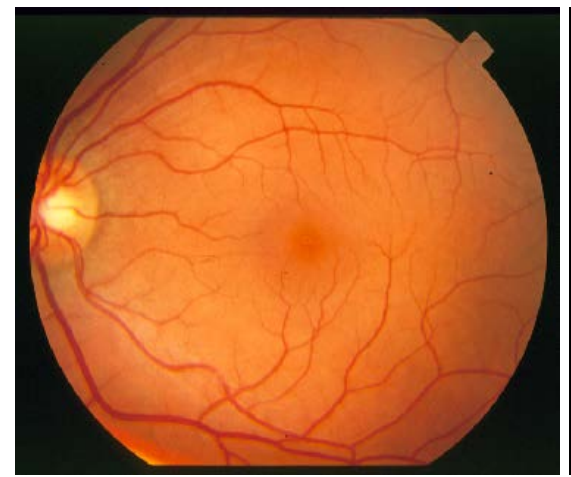

(a)

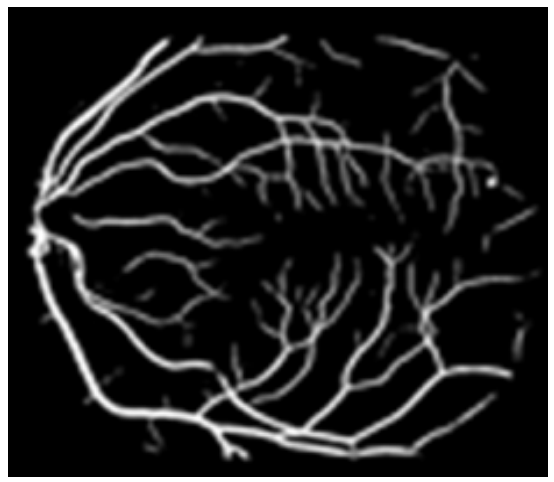

(d)

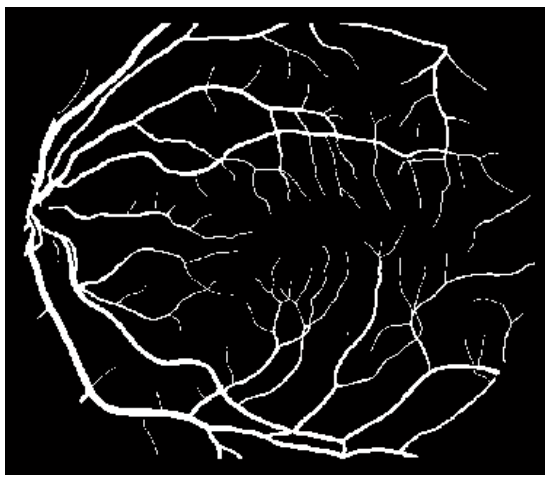

(b)

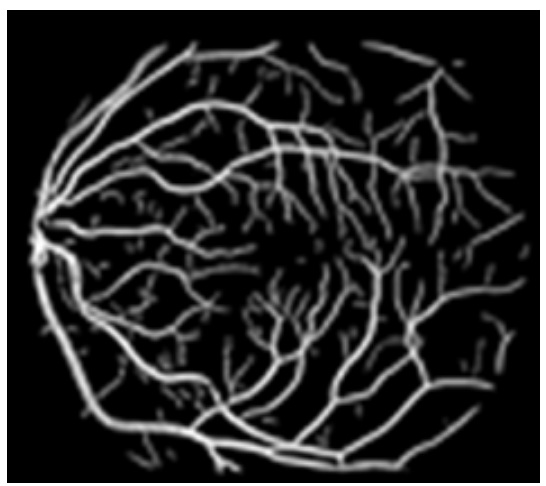

(e)

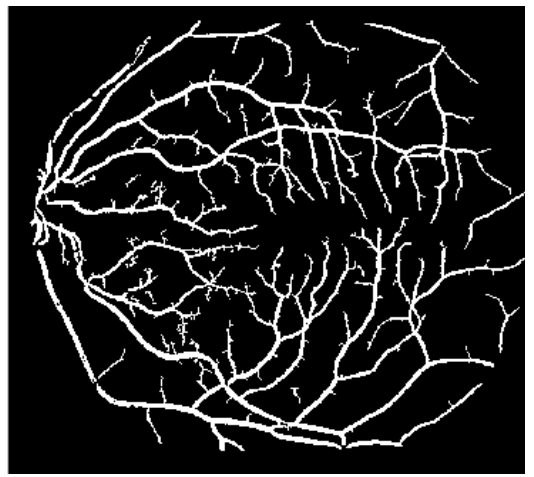

(c)

Fig. 3 Results of the different approaches applied to the publicy STARE dataset. (a) Original image. (b) Manual method. (c) Proposed mehtod. (d) Zhang et al.. (e) Dai et al.. 


\section{Experiments}

The publicly fundus image dataset STARE [7] is sued for verify the validity and feasibility of the proposed method. Fig. 3 compares the different vessel segmentation results with different methods. Fig. 3 (a) shows the original retinal color image, Fig. 3 (b) shows the manual segmentation result, Fig. 3 (c) shows the segmentation result of the proposed method, Fig. 3 (d) shows the segmentation result of Zhang et al. [8], Fig. 3 (e) shows the segmentation result of Dai et al. [9].

\section{Conclusion}

This paper proposed a retinal segmentation mehtod based on top-hat transformation, this approach include three steps: preprocessing, vessel enhancement and vessel segmentation. Then this method is tested by the publicy database STARE. the results of the experiment show that segmentation effect of the proposed method is better than other methods.

\section{Acknowledgements}

Thanks JJ Staal, Hoover and their colleagues for providing their databases. This work is supported by the National Natural Science Foundation of China (61202131, 61304255,11301524, and 11471307), the Academician Special Project of Chongqing Basic and Frontier Research Program (cstc2015jcyjys40001), the West Light Foundation of Chinese Academy of Sciences, the Scientific and Technological Project of Chongqing Municipal Education Commission (KJ1401118, KJ1501120 and KJ131225), the Scientific and Technological Project of Yongchuan District under (Ycstc2013ad5001).

\section{References}

[1] Roychowdhury, S., D.D. Koozekanani and K.K. Parhi, Iterative Vessel Segmentation of Fundus Images. IEEE Transactions on Biomedical Engineering, 2015. 62(7): p. 1738-1749.

[2] Azzopardi, G., et al., Trainable COSFIRE filters for vessel delineation with application to retinal images. Medical Image Analysis, 2015. 19(1): p. 46-57.

[3] Chaudhuri, S., et al., Detection of blood vessels in retinal images using two-dimensional matched filters. IEEE Transactions on Medical Imaging, 1989. 8(3): p. 263-269.

[4] Soares, J.V.B., et al., Retinal vessel segmentation using the 2-D Gabor wavelet and supervised classification. IEEE Transactions on Medical Imaging, 2006. 25(9): p. 1214-1222.

[5] Marin, D., et al., A new supervised method for blood vessel segmentation in retinal images by using gray-level and moment invariants-based features. IEEE Transactions on Medical Imaging, 2011. 30(1): p. 146-58.

[6] You, X., et al., Segmentation of retinal blood vessels using the radial projection and semi-supervised approach. Pattern Recognition, 2011. 44(10 - 11): p. 2314-2324.

[7] Al-Rawi, M., M. Qutaishat and M. Arrar, An improved matched filter for blood vessel detection of digital retinal images. Computers in Biology \& Medicine, 2007. 37(2): p. 262-267.

[8] Zhang, B., et al., Retinal vessel extraction by matched filter with first-order derivative of Gaussian. Computers in Biology and Medicine, 2010. 40(4): p. 438-445.

[9] Dai, P., et al., A New Approach to Segment Both Main and Peripheral Retinal Vessels Based on Gray-Voting and Gaussian Mixture Model. PLoS ONE, 2015. 10(6): p. e0127748. 